\title{
Numerical simulation and validation of dilute turbulent gas-particle flow with inelastic collisions and turbulence modulation
}

\author{
Aldo Benavides*, Berend van Wachem \\ Applied Mechanics \\ Chalmers University of Technology \\ SE-41296 Gothenburg Sweden
}

\begin{abstract}
This work describes a theoretical and numerical study of turbulent gas-particle flows in the Eulerian framework. The equations describing the flow are derived employing Favre averaging. The closures required for the equations describing the particulate phase are derived from the kinetic theory of granular flow. The kinetic theory proposed originally is extended to incorporate the effects of the continuous fluid on the particulate phase behavior. Models describing the coupling between the continuous phase kinetic energy and particulate phase granular temperature are derived, discussed, and their effect on the flow predictions is shown.

The derived models are validated with benchmark experimental results of a fully developed turbulent gas-solid flow in a vertical pipe. The effect of the models describing the influence of turbulence on the particle motion as well as the turbulence modulation due to the presence of particles is analyzed and discussed.
\end{abstract}

Keywords: gas-solid flow; turbulence modulation; kinetic theory of granular flow.

\section{Introduction}

Turbulent gas-solid flows are found in many industrial applications such as cyclone separators, jet mills, fluidized beds, inhalers, nuclear reactor cooling systems, pneumatic transport of powders and pulverized-coal combustors. They also occur in nature, e.g., desert sand storms, pollen in air and dispersion of pollutants. For a reliable understanding of turbulent gas-solid flow

* Corresponding author.

Email address: benavide@chalmers.se (Aldo Benavides). 
phenomena, adequate models are needed. Such modeling will improve the design and optimization of processes dealing with this flow type. To successfully simulate a gas-solid flow system, it is necessary to account for particle-particle collisions as well as gas-particle interactions, mainly the effect of the gas-phase turbulence on the particle motion and vice versa. Furthermore, the momentum and energy transfer due to particle collisions needs to be properly formulated according to the kinetic theory of granular flow.

Gas-solid flow models can be divided into two groups, Lagrangian and Eulerian models [1]. Lagrangian, or discrete particle models, trace the path of individual particles on their way through the flow field by the equation of motion, that is, Newton's second law. However, actual computer capabilities impose a restriction on the number of particles that can be tracked, which makes this model unfeasible for many industrial-size problems. Additionally, forces from the gas phase acting on each particle are difficult to model. On the other hand, the Eulerian or two-fluid model handles the solid-phase as a continuum in which the motion of individual particles is averaged such that mean equations are solved for both gas and solid phases and coupled through interfacial transfer terms. Thus, the problem of tracking individual particles is avoided, but closure relations are required for the arising correlations within each phase and between phases. Moreover, the extent to which momentum and energy are transferred among the phases determines the degree of coupling. The latter approach is the prevailing method for practical applications and the one described in this work.

Depending on the way that momentum and energy is transferred in the solid phase, a particular gas-solid flow can be classified into three regimes [2]: the macro-viscous regime, where the disturbance flow field of one particle affects the motion of the surrounding particles; the grain-inertia regime which is dominated by direct collisions between particles; and the transitional regime which is controlled by both mechanisms. Several forces might influence the motion of particles; however the drag force exerted by the carrier gas can be regarded as the most important one. Actually, it might be the only driving force that sustains the solid-phase flow through interfacial momentum transfer. Other forces that can be present are gravity, the Archimedes force, history or Basset force, lift due to fluid shearing motion and particle rotation, friction due to particle-particle contact and dispersion due to the gas turbulence.

The kinetic theory of dry granular flow, derived by Lun et al. [3], was among the first attempts to obtain hydrodynamic equations as well as appropriate constitutive relations for the solid phase. Similarly, Jenkis et al. [4] employed Grad's 13 moment method in order to obtain the single-particle velocity distribution function (PDF) which allows solving the integral forms for the collisional rate of change of any particle property. However, the effects of the interstitial fluid were not taken into account in these earlier works. Further 
investigation on how the interstitial turbulent gas modifies the constitutive relations of the solid phase was carried out by Reeks [5], Simonin [6], Zhang and Reese [7] and Lun and Savage [8]. Peirano and Leckner [9] derived suitable relations for the solid-phase stress tensor and fluctuating energy flux under the Eulerian framework, which incorporate the effect of gas turbulence on the random motion of particles.

For gas-solid flows at high Reynolds numbers, the gas turbulence is expected to have a noticeable effect on the momentum and energy transfer between both phases [10]. In fact, its main effect is thought to be the dispersion of particles throughout the flow field, having a distinct influence on the solid phase distribution. As the particle diameter can be somewhere in between the Kolmogorov length scale and the integral length scale of the gas turbulence, the presence of particles may also modulate the turbulence structure in the gas phase [8]. As pointed out by Peirano and Leckner [9], the vortex shedding and work done by eddies are thought to be the dominant mechanisms for turbulence modulation in gas-solid flows. An alternative derivation of the two-fluid model equations including fluid-solid interaction was developed by Simonin [6] based also on particle PDF transport equations. In his approach, fluid turbulence correlated with the particulate phase is modeled using Langevin equation [11].

Although dilute gas-solid suspensions have been extensively studied, there is still no general agreement on the transport coefficients and constitutive relations for the two-fluid model. The gas phase turbulence has usually been modeled by a standard $k-\epsilon$ model with additional terms which account for the gas-solid interaction. However, these adjustments to the gas-solid two-phase flow model are based on heuristic models which may not reflect the appropriate interaction in most cases. Motivating the form of such equations has a central role in the theory of gas-solid two-phase flows and it represents a challenge to model it. In this work, a consistent model will be based upon the decomposed and averaged governing equations for a two-fluid system.

The main objective of the present work is to acquire an adequate understanding of the general physical phenomena involved in gas-solid flows with emphasis on the interaction between particle motion and gas phase turbulence. It also aims to provide the fundamentals for numerical calculations of turbulent gas-solid flows. Turbulent gas-solid flow in a vertical pipe is chosen as the test case for validation of two-fluid model performance. The simulation results are compared with existing experimental data [12] obtained from laser Doppler velocimetry (LDV). 


\section{Continuum equations for a two-fluid system}

Derivations of Eulerian forms of conservation of mass, momentum and energy for a multi-fluid system are similar to those for a single phase flow [13]. In this paper, equations are expressed using tensor notation where is appropriate. Instantaneous quantities are denoted by a tilde, i.e. $\tilde{f}$. The corresponding upper-case letter, $F$, indicates a mean quantity (obtained from a weightedaverage) whereas time-averaged quantities are denoted by an overbar, $\bar{F}$. In addition, fluctuations are represented by a single lower-case letter, i.e. $f$ (exceptions are instantaneous particle velocity, $\boldsymbol{c}$, and gravity, g).

Attention is restricted to constant density constituents, $\tilde{\rho}_{l}(\boldsymbol{x}, t)=\rho_{l}$, which is usually a good assumption in engineering gas-solid flows. If it is further assumed that there is no source of mass due to phase change or chemical reaction, the instantaneous $l$-phase continuity equation can be written as

$$
\frac{\partial \tilde{\phi}_{l}}{\partial t}+\frac{\partial}{\partial x^{i}}\left(\tilde{\phi}_{l} \tilde{u}_{l}^{i}\right)=0
$$

where $\tilde{\phi}_{l}$ and $\tilde{u}_{l}^{i}$ are the volume fraction and $i$-velocity component for phase $l$, respectively. Besides, conservation of total volume implies

$$
\sum_{l=1}^{N_{\text {phases }}} \tilde{\phi}_{l}=1
$$

So, equations (1) and (2) can be combined to obtain a differential form for the conservation of total volume

$$
\sum_{l=1}^{N_{\text {phases }}} \frac{\partial}{\partial x^{i}}\left(\tilde{\phi}_{l} \tilde{u}_{l}^{i}\right)=0
$$

which is the incompressible flow condition in multiphase flow.

The momentum equation for phase $l$ in direction $i$ can be expressed as

$$
\frac{\partial}{\partial t}\left(\rho_{l} \tilde{\phi}_{l} \tilde{u}_{l}^{i}\right)+\frac{\partial}{\partial x^{j}}\left(\rho_{l} \tilde{\phi}_{l} \tilde{u}_{l}^{j} \tilde{u}_{l}^{i}\right)=\frac{\partial}{\partial x^{j}}\left(\tilde{\phi}_{l} \tilde{\mathcal{T}}_{l}^{i j}\right)-\tilde{\phi}_{l} \frac{\partial \tilde{p}}{\partial x^{i}}+\rho_{l} \tilde{\phi}_{l} \tilde{b}_{l}^{i}+\tilde{m}_{l}^{i}
$$

where $\tilde{\mathcal{T}}_{l}^{i j}$ is the stress tensor relative to phase $l ; \tilde{p}$ is the shared pressure field; $\tilde{b}_{l}^{i}$ is the force per unit mass due to external sources, and $\tilde{m}_{l}^{i}$ stands for the (instantaneous) force on phase $l$ due to interaction with other constituents or, in other words, a momentum exchange term. Thus, momentum conservation requires that

$$
\sum_{l=1}^{N_{\text {phases }}} \tilde{m}_{l}^{i}=0
$$


By performing Reynolds decomposition on instantaneous quantities and averaging, mean transport equations can be obtained. Any flow variable can be decomposed into a mean part and a fluctuating part as follows

$$
\begin{aligned}
& \tilde{\phi}_{l}=\Phi_{l}+\phi_{l} \\
& \tilde{u}_{l}^{i}=\bar{U}_{l}^{i}+u_{l}^{i}
\end{aligned}
$$

As $\Phi_{l}=\bar{\phi}_{l}$, then $\overline{\phi_{l}}=0$ which is also true for the time-average of any fluctuating quantity. It should be noted that, by definition, the volume fraction is a volume averaged quantity, so the meaning of $\phi_{l}$ represents fluctuations at a larger scale, than the scale employed to define the volume fraction. Such fluctuations thus represent, for instance, particle clusters.

In order to reduce the number of terms appearing in the Reynolds-averaged like transport equations, a weighted-average or Favre-average is employed. For instance, the Favre-averaged velocity becomes

$$
U_{l}^{i}=\frac{\overline{\tilde{\phi}_{l} \tilde{u}_{l}^{i}}}{\Phi_{l}}=\bar{U}_{l}^{i}+\frac{\overline{\phi_{l} u_{l}^{i}}}{\Phi_{l}}
$$

Favre-averaged values are indeed obtained from LDA measurements (laser Doppler anemometry) when sampling flow quantities such as velocity, i.e., the probe starts sampling as soon as phase $l$ is detected.

Averaging equation (1) results in the $l$-phase continuity equation for the mean motion

$$
\frac{\partial \Phi_{l}}{\partial t}+\frac{\partial}{\partial x^{i}}\left(\Phi_{l} U_{l}^{i}\right)=0
$$

If time-averaging were used, an additional source term due to the correlation between fluctuations in volume fraction and velocity components would appear in the continuity equation [14].

Similarly, equation (4) can be decomposed and averaged in order to obtain the $l$-phase momentum conservation equation for the mean motion

$$
\begin{aligned}
\frac{\partial}{\partial t}\left(\rho_{l} \Phi_{l} U_{l}^{i}\right)+\frac{\partial}{\partial x^{j}}\left(\rho_{l} \Phi_{l} U_{l}^{j} U_{l}^{i}\right) & =\frac{\partial}{\partial x^{j}}\left[\Phi_{l}\left(\mathcal{T}_{l}^{i j}+R_{l}^{i j}\right)-\rho_{l} \overline{\phi_{l} u_{l}^{i} u_{l}^{j}}\right]+ \\
& -\Phi_{l} \frac{\partial P}{\partial x^{i}}+\rho_{l} \Phi_{l} B_{l}^{i}+M_{l}^{i}
\end{aligned}
$$

where the Reynolds stress tensor, $R_{l}^{i j}=-\rho_{l} \overline{u_{l}^{i} u_{l}^{j}}$, can be identified on the right hand side together with the triple correlation between fluctuating velocities and volume fraction, $\overline{\phi_{l} u_{l}^{i} u_{l}^{j}}$. These are direct results from Favre-averaging. 
A transport equation for the fluctuations, $u_{l}^{i}$, can be obtained by subtracting the mean momentum equation (9) from equation (4) as described in [14]. Then, an equation for the $l$-phase fluctuating kinetic energy, $k_{l}=\frac{1}{2} \overline{u_{l}^{i} u_{l}^{i}}=\frac{1}{2} \overline{u_{l}^{2}}$, can be derived by multiplying the resulting equation for the fluctuating motion by $u_{l}^{i}$ and averaging. A general form for the transport equation of turbulent kinetic energy relative to phase $l$ can be written as

$$
\begin{aligned}
\frac{\partial}{\partial t}\left(\rho_{l} \Phi_{l} k_{l}\right)+\frac{\partial}{\partial x^{i}}\left(\rho_{l} \Phi_{l} U_{l}^{i} k_{l}\right)+\mathcal{D}_{l}^{t}= & -\frac{\partial}{\partial x^{j}}\left(\Phi_{l} \mathcal{J}_{l}^{j}\right)+ \\
& \overline{\rho_{l} \frac{1}{2} u_{l}^{2} \frac{\partial}{\partial x^{j}}\left(u_{l}^{j} \Phi_{l}\right)}+\Phi_{l} \overline{p \frac{\partial u_{l}^{i}}{\partial x^{i}}}+ \\
& \mathcal{P}_{l}-\Phi_{l} \epsilon_{l}+\rho_{l} \Phi_{l} \overline{u_{l}^{i} b_{l}^{i}}+\overline{u_{l}^{i} m_{l}^{i}}
\end{aligned}
$$

The above equation provides the basis for modeling the energy associated with the fluctuating motion on each phase. The role of each term in (10) can be clarified as follows. There is an extra-transient term on the left hand side of (10), $\mathcal{D}_{l}^{t}$, which accounts for changes in time of correlations between fluctuations in volume fraction and velocity, i.e $\overline{\phi_{l} u_{l}^{i}}$. Similar terms are neglected in [14] so that $\frac{\partial}{\partial t}\left(\Phi_{l} k_{l}\right)$ is considered as the dominant transient term. The first term in brackets on the right hand side represents the flux of fluctuating kinetic energy due to pressure fluctuations, turbulent advective transport and the stress tensor, respectively

$$
\mathcal{J}_{l}^{j}=\overline{u_{l}^{j} p}+\rho_{l} \overline{\frac{1}{2} u_{l}^{2} u_{l}^{j}}-\overline{\tau_{l}^{i j} u_{l}^{i}}
$$

Again, contributions to the flux $\mathcal{J}_{l}^{j}$ from correlations involving the fluctuating volume fraction are not considered. The second term on the right hand side of (10) completes the diffusion due to turbulence. The pressure velocitydivergence correlation describes the transfer of energy between the turbulence and the inner energy of the fluid. Such energy exchange is nearly always negligible for gas-solid flows of interest [9]. The term $\mathcal{P}_{l}$ accounts for the rate of production of turbulence kinetic energy from the mean flow

$$
\mathcal{P}_{l}=R_{l}^{i j} \frac{\partial}{\partial x^{j}}\left(\Phi_{l} U_{l}^{i}\right)-\rho_{l} \Phi_{l} U_{l}^{i} \overline{u_{l}^{i} \frac{\partial u_{l}^{j}}{\partial x^{j}}}
$$

where the contribution to the rate of production from the second term in (12) is thought to be much smaller than the one from the mean flow gradients. Next term, $\epsilon_{l}$, stands for the rate of dissipation of turbulence energy

$$
\epsilon_{l}=\overline{\tau_{l}^{i j} \frac{\partial u_{l}^{i}}{\partial x^{j}}}
$$

It should be noticed that extra-production and extra-dissipation terms due to correlations involving the fluctuating volume fraction are not considered in 
Table 1

General averaged transport equations for a multifluid system.

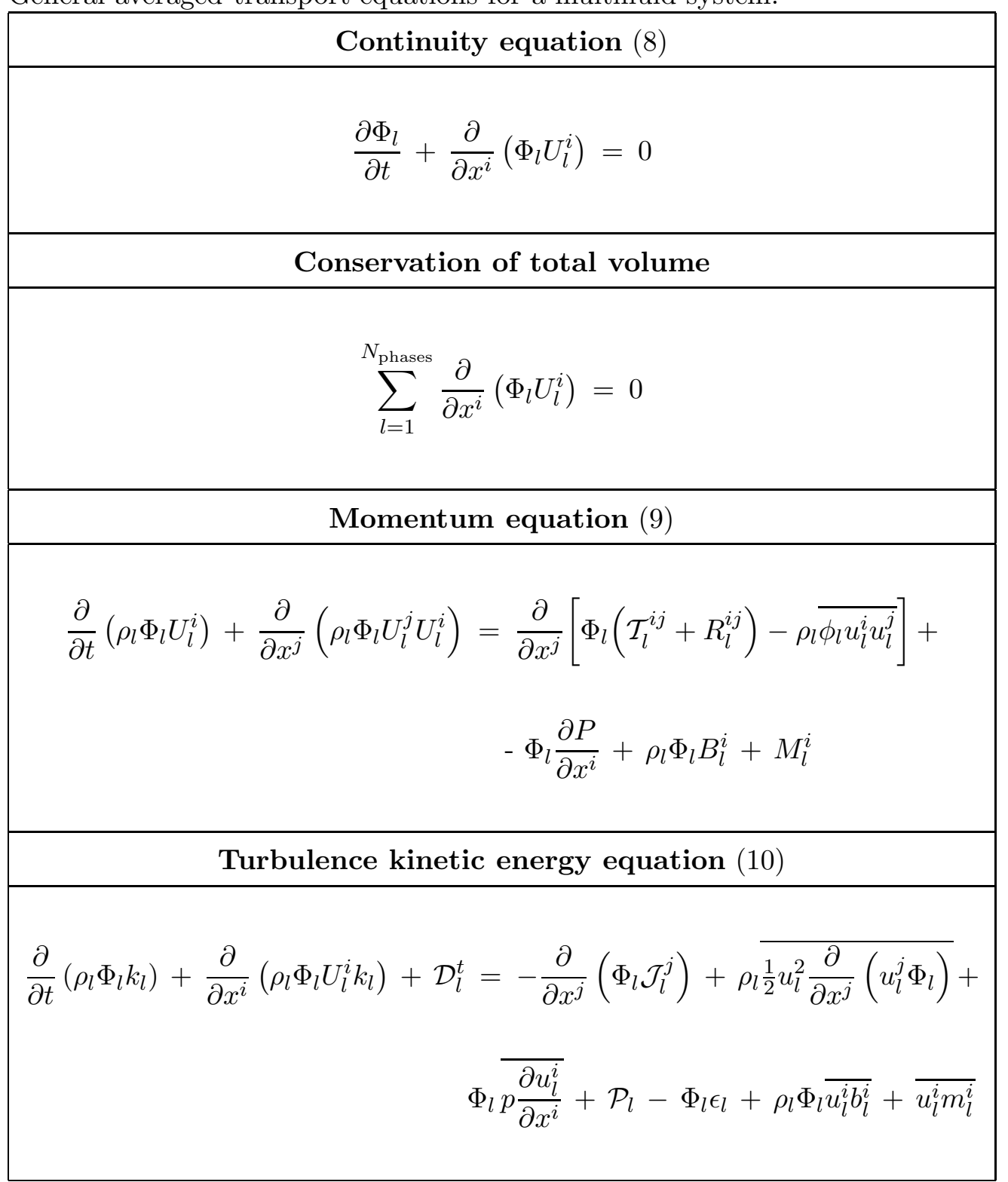

(12) and (13). The second to last term in equation (10) is the work done by the external forces and the last term represents the interfacial work. Thus, the interaction among fluids is expressed by the (averaged) momentum exchange term in (9) and the interfacial work in (10). Moreover, it is evident that the presence of more than one phase modifies the stress tensor and turbulence kinetic energy flux. In the next sections, attention will be focused on examining plausible closure relations for these interaction terms regarding a gas-solid flow as well as understanding the effect of each phase on the constitutive relations of the other. The set of mean transport equations for a two-fluid system is summarized in Table 1. 


\section{Transport equations for gas-solid flow}

Equations (8), (9) and (10) combined with conditions (2) and (5) represent a set of general equations for a multi-fluid system. By examining the nature of a given constituent, the form for the $l$-phase stress tensor can be formulated. For a fluid (gas-phase) the tensor $\mathcal{T}_{l}^{i j}$ represents the viscous stress tensor which is proportional to the strain-rate tensor, i.e., a Newtonian fluid

$$
S_{l}^{i j}=\frac{1}{2}\left[\frac{\partial U_{l}^{i}}{\partial x^{j}}+\frac{\partial U_{l}^{j}}{\partial x^{i}}\right]
$$

The effect of gravity is included into the external force term, $B_{l}^{i}$, in (9). Thus, the momentum equation for the gas-phase mean motion becomes

$$
\begin{aligned}
\frac{\partial}{\partial t}\left(\rho_{f} \Phi_{f} U_{f}^{i}\right)+\frac{\partial}{\partial x^{j}}\left(\rho_{f} \Phi_{f} U_{f}^{j} U_{f}^{i}\right)= & \frac{\partial}{\partial x^{j}}\left[\Phi_{f}\left(\mathcal{T}_{f}^{i j}+R_{f}^{i j}\right)\right]+ \\
& -\Phi_{f} \frac{\partial P}{\partial x^{i}}+\rho_{f} \Phi_{f} g^{i}+M_{f}^{i}
\end{aligned}
$$

where subscript $f$ holds for the carrier phase (gas). The constitutive relations for viscous stress tensor, $\mathcal{T}_{f}^{i j}$, and the closure for Reynolds stresses, $R_{f}^{i j}$, are given in the next section. It should be noted that correlations among velocity fluctuations and volume fraction, $\overline{\phi_{f} u_{f}^{i} u_{f}^{j}}$, are not considered. The evaluation of this term would require an additional transport equation or a closure model which, for instance, could depend on gradients of $\overline{\phi_{f} u_{f}^{i}}[14]$. This is not regarded in the current approach.

Similarly, the momentum equation for the dispersed phase (denoted by subscript $s$ ) can be written as

$$
\begin{aligned}
\frac{\partial}{\partial t}\left(\rho_{s} \Phi_{s} U_{s}^{i}\right)+\frac{\partial}{\partial x^{j}}\left(\rho_{s} \Phi_{s} U_{s}^{j} U_{s}^{i}\right)= & \frac{\partial}{\partial x^{j}}\left[\Phi_{s}\left(R_{s}^{i j}+\mathcal{T}_{s}^{i j}\right)\right]+ \\
& -\Phi_{s} \frac{\partial P}{\partial x^{i}}+\rho_{s} \Phi_{s} g^{i}+M_{s}^{i}
\end{aligned}
$$

where the first term between brackets on the right hand side, $R_{s}^{i j}$ stands for the kinetic stress tensor (second moment in solid velocity fluctuations) and the second term, $\mathcal{T}_{s}^{i j}$, is the collisional stress tensor in the particulate phase. Expressions for these terms are derived from the kinetic theory of dry granular flow in [4] and modifications to incorporate the interstitial gas effect on the constitutive relations are described in [9]. The latter constitutive equations are employed here to provide the closures for the dispersed phase. Finally, the 
gas-phase fluctuating kinetic energy can be written as

$$
\frac{\partial}{\partial t}\left(\rho_{f} \Phi_{f} k_{f}\right)+\frac{\partial}{\partial x^{i}}\left(\rho_{f} \Phi_{f} U_{f}^{i} k_{f}\right)=-\frac{\partial}{\partial x^{j}}\left[\Phi_{f} \mathcal{J}_{f}^{j}\right]+\mathcal{P}_{f}-\Phi_{f} \epsilon_{f}+\overline{u_{f}^{i} m_{f}^{i}}
$$

where the flux $\mathcal{J}_{f}^{j}$ collects the diffusive effect of turbulence due to the advective action of the velocity fluctuations, pressure transport and viscous flux of $k_{f}$, as it can be interpreted from (11). Furthermore, the pressure velocitydivergence term and work done by fluctuating external forces are disregarded. The production rate of $k_{f}$ becomes

$$
\mathcal{P}_{f}=R_{f}^{i j} \frac{\partial}{\partial x^{j}}\left(\Phi_{f} U_{f}^{i}\right)
$$

so production of kinetic energy within the carrier phase is only due to the action of Reynolds stresses. The rate of dissipation, $\epsilon_{f}$, requires a closure model or an additional (modeled) transport equation.

On the other hand, the fluctuating kinetic energy of the particulate phase can be recast in terms of the so-called granular temperature, $\Theta_{s}=\frac{1}{3} \overline{u_{s}^{i} u_{s}^{i}}$. According to the current approach, the transport equation for granular temperature can be expressed as

$$
\frac{3}{2}\left[\frac{\partial}{\partial t}\left(\rho_{s} \Phi_{s} \Theta_{s}\right)+\frac{\partial}{\partial x^{i}}\left(\rho_{s} \Phi_{s} U_{s}^{i} \Theta_{s}\right)\right]=-\frac{\partial}{\partial x^{j}}\left[\Phi_{s} \mathcal{J}_{s}^{j}\right]+\mathcal{P}_{s}-\Phi_{s} \epsilon_{s}+\overline{u_{s}^{i} m_{s}^{i}}
$$

where the production term $\mathcal{P}_{s}$, in the case of a dispersed phase, is due to both kinetic and collisional stresses. Furthermore, the random motion of particles and direct particle-particle interaction (collisions) give rise to an energy flux, $\mathcal{J}_{s}^{j}$, analogous to the general flux term in (11). The rate of energy dissipation, $\epsilon_{s}$, represents energy loss in collisions which also needs to be accounted for. Before going on with the description of the constitutive relations within each phase and the closure models, a comparison is carried out between the current derivation of the solid-phase transport equations and the kinetic theory approach. It is necessary to introduce this theory in order to obtain explicit expressions for the kinetic and collisional stress tensors, energy flux and rate of collisional energy dissipation in the solid-phase.

\subsection{Kinetic theory of granular flow}

The analogy with the Chapman-Enskog kinetic theory for dense gases [15] and other statistical methods developed for gases have been commonly used in the analysis of granular flow. It is the prevailing theory (if not the only one) employed to accurately describe the particulate phase without the necessity of empirical parameters. A brief outline of this theory is presented here as well 
Table 2

Gas-solid two-phase flow transport equations

\begin{tabular}{|c|}
\hline Solid-phase continuity equation \\
\hline$\frac{\partial \Phi_{s}}{\partial t}+\frac{\partial}{\partial x^{i}}\left(\Phi_{s} U_{s}^{i}\right)=0$ \\
\hline Conservation of total volume \\
\hline$\Phi_{s}+\Phi_{f}=1 \quad$ or $\quad \frac{\partial}{\partial x^{i}}\left(\Phi_{s} U_{s}^{i}+\Phi_{f} U_{f}^{i}\right)=0$ \\
\hline Solid-phase momentum equation (16) \\
\hline $\begin{array}{c}\frac{\partial}{\partial t}\left(\rho_{s} \Phi_{s} U_{s}^{i}\right)+\frac{\partial}{\partial x^{j}}\left(\rho_{s} \Phi_{s} U_{s}^{j} U_{s}^{i}\right)= \\
\frac{\partial}{\partial x^{j}}\left[\Phi_{s}\left(R_{s}^{i j}+\mathcal{T}_{s}^{i j}\right)\right]-\Phi_{s} \frac{\partial P}{\partial x^{i}}+ \\
\rho_{s} \Phi_{s} g^{i}+M_{s}^{i}\end{array}$ \\
\hline Granular Temperature equation (19) \\
\hline$\frac{3}{2}\left[\frac{\partial}{\partial t}\left(\rho_{s} \Phi_{s} \Theta_{s}\right)+\frac{\partial}{\partial x^{i}}\left(\rho_{s} \Phi_{s} U_{s}^{i} \Theta_{s}\right)\right]=-\frac{\partial}{\partial x^{j}}\left[\Phi_{s} \mathcal{J}_{s}^{j}\right]+\mathcal{P}_{s}-\Phi_{s} \epsilon_{s}+\overline{u_{s}^{i} m_{s}^{i}}$ \\
\hline Gas-phase momentum equation (15) \\
\hline$\frac{\partial}{\partial t}\left(\rho_{f} \Phi_{f} U_{f}^{i}\right)+\frac{\partial}{\partial x^{j}}\left(\rho_{f} \Phi_{f} U_{f}^{j} U_{f}^{i}\right)=\frac{\partial}{\partial x^{j}}\left[\Phi_{f}\left(\mathcal{T}_{f}^{i j}+R_{f}^{i j}\right)\right]-\Phi_{f} \frac{\partial P}{\partial x^{i}}+M_{f}^{i}$ \\
\hline Gas-phase turbulent kinetic energy $(17)$ \\
\hline$\frac{\partial}{\partial t}\left(\rho_{f} \Phi_{f} k_{f}\right)+\frac{\partial}{\partial x^{i}}\left(\rho_{f} \Phi_{f} U_{f}^{i} k_{f}\right)=-\frac{\partial}{\partial x^{j}}\left[\Phi_{f} \mathcal{J}_{f}^{j}\right]+\mathcal{P}_{f}-\Phi_{f} \epsilon_{f}+\overline{u_{f}^{i} m_{f}^{i}}$ \\
\hline
\end{tabular}

as a comparison between the solid-phase transport equations already derived and the results of the kinetic theory of granular flow. The theory applies in the case of flow of uniform spherical particles if it is assumed that collisions occur instantaneously between pairs of smooth spheres in translational motion. As kinetic energy of the particles is lost in collisions between pairs of grains, their inelasticity is taken into account through the coefficient of restitution, 
$e_{s}\left(0 \leq e_{s} \leq 1\right)$. The distribution of particle velocities is characterized by the single particle velocity probability density function $(\mathrm{PDF}) f^{(1)}(\boldsymbol{c}, \boldsymbol{x}, t)$ which is defined such that the probable number of particles which at time $t$ are situated in the volume element $(\boldsymbol{x}, \boldsymbol{x}+d \boldsymbol{x})$ and have velocities lying in the range $(\boldsymbol{c}, \boldsymbol{c}+d \boldsymbol{c})$ is $f^{(1)}(\boldsymbol{c}, \boldsymbol{x}, t) d \boldsymbol{c} d \boldsymbol{x}$. The change in $f^{(1)}$ over a time interval can be caused by direct collision between pairs of particles (though their motion may be influenced by the presence of an interstitial medium). Thus the net change of $f^{(1)}$ during $d t$ is governed by

$$
\frac{\partial f^{(1)}}{\partial t}+c^{j} \frac{\partial f^{(1)}}{\partial x^{j}}+\tilde{a}^{j} \frac{\partial f^{(1)}}{\partial c^{j}}=\left(\frac{\partial f^{(1)}}{\partial t}\right)_{C}
$$

which is known as the Boltzmann equation. The right hand side stands for the rate of change of $f^{(1)}$ due to direct collisions between pairs of particles. The last term on the left hand side represents any change on the PDF due to external forces (per unit mass), $\tilde{a}^{j}$, which comprises gravity effects and particle interaction with a surrounding medium. Besides, the PDF allows to define the ensemble average of any particle property, $\psi(\boldsymbol{c}, \boldsymbol{x}, t)$, as

$$
\langle\psi(\boldsymbol{x}, t)\rangle=\frac{1}{n} \int \psi(\boldsymbol{c}, \boldsymbol{x}, t) f^{(1)}(\boldsymbol{c}, \boldsymbol{x}, t) d \boldsymbol{c}
$$

where $n$ is the number density of particles defined by integrating the PDF over the whole velocity-space. Thus, any mean quantity can be obtained from (21), i.e. $U_{s}^{i}=\left\langle c^{i}\right\rangle$ and $u_{s}^{i}=c^{i}-U_{s}^{i}$, which also allows to keep the notation employed so far, but regarding mean quantities in the dispersed phase as defined by (21). Following the derivations outlined in [1], a transport equation for any averaged particle property can be obtained after multiplying equation (20) by the particle property $\psi$ and integrating over the velocity space. The general transport equation is known as Enskog equation

$$
\begin{array}{r}
\frac{D(n\langle\psi\rangle)}{D t}+n\langle\psi\rangle \frac{\partial U_{s}^{j}}{\partial x^{j}}+\frac{\partial}{\partial x^{j}}\left(n\left\langle\psi u_{s}^{j}\right\rangle\right)+n \frac{D U_{s}^{j}}{D t}\left\langle\frac{\partial \psi}{\partial u_{s}^{j}}\right\rangle= \\
n\langle D \psi\rangle-n\left\langle u_{s}^{j} \frac{\partial \psi}{\partial u_{s}^{k}}\right\rangle \frac{\partial U_{s}^{k}}{\partial x^{j}}+n \mathbb{C}(\psi)
\end{array}
$$

where $D \psi$ is given by

$$
D \psi=\frac{D \psi}{D t}+u_{s}^{i} \frac{\partial \psi}{\partial x^{i}}+a^{i} \frac{\partial \psi}{\partial u_{s}^{i}}
$$

and the operator $\frac{D}{D t}$ stands for the material derivative following the mean flow. The right hand side of (20) times $\psi$ integrates to $n \mathbb{C}(\psi)$, which stands for the collisional rate of change of the particle property per unit volume. The statistics of binary collisions is characterized by the pair probability distribution 
function, $f^{(2)}\left(\boldsymbol{c}_{1}, \boldsymbol{x}_{1}, \boldsymbol{c}_{2}, \boldsymbol{x}_{2}, t\right)$. Based on the assumption of molecular chaos [15] the pair probability distribution function can be related to the PDF of each colliding particle, assuming that the particle velocities prior to collision are uncorrelated with each other. The pair distribution function is further adjusted by a radial distribution function at contact, $g_{0}$, which intends to reduce the volume in which the center of any particle can lie, thus ensuring the nonoverlapping of moving particles and increasing the probability of a collision. Several expressions for $g_{0}$ have been proposed throughout the development of kinetic theory. A typical expression is found in Table 3, as suggested by Sinclair and Jackson [1]. The integral of the change of $\psi$ due to a binary collisions can be recast into a more suitable form after performing a Taylor series expansion of $f^{(2)}$ about the point of contact between colliding particles as outlined in [4]. Thus, the collisional rate of change of any particle property can be written as

$$
n \mathbb{C}(\psi)=\chi_{s}(\psi)-\frac{\partial \Theta_{s}^{j}(\psi)}{\partial x^{j}}-\frac{\partial U_{s}^{j}}{\partial x^{i}} \Theta_{s}^{i}\left(\frac{\partial \psi}{\partial u_{s}^{j}}\right)
$$

The first term on the right hand side is the collisional source (or sink) term, $\chi_{s}(\psi)$, which represents the loss of $\psi$ due to inelastic collisions and the collisional flux term, $\Theta_{s}^{i}(\psi)$, which is responsible for the transfer of $\psi$ during a collision. The derivative in parenthesis is an argument to the tensor $\Theta_{s}^{i}$. The source and flux terms are defined by integrals which depend on $f^{(2)}$. These integrals can be solved analytically by regarding some linear theory simplifications together with Grad's method. The derivation is thoroughly described in [4].

Conservation equations of mass, momentum and fluctuating kinetic energy for the solid-phase can be obtained after substituting $\psi$ by $\mathrm{m}, \mathrm{m} u_{s}^{i}$, and $\frac{1}{2} \mathrm{~m} u_{s}^{2}$, respectively in equation (22), where $\mathrm{m}$ is the mass of a single spherical particle. Conservation of mass in the solid-phase has the form of equation (8) provided that $\mathrm{m} n=\Phi_{s} \rho_{s}$. The same applies for momentum and fluctuating kinetic energy. The external force per unit mass matches the last three terms on the right hand side of equation (16) only if

$$
\tilde{a}^{i}=-\frac{1}{\rho_{s}} \frac{\partial \tilde{p}}{\partial x^{i}}+\mathrm{g}^{i}+\frac{1}{\mathrm{~m} n} \tilde{m}_{s}^{i}
$$

where the force exerted by the pressure gradient on the dispersed phase can be regarded as an Archimedes-like force. Moreover, the only contribution from the collisional rate of change of momentum in (24) is the flux of $m u_{s}^{i}$, or in other words, the divergence of the collisional stress tensor, $\Theta_{s}^{j}\left(\mathrm{~m} u_{s}^{i}\right)$, which matches $\Phi_{s} \mathcal{T}_{s}^{i j}$ in equation (16). Furthermore, the kinetic stress tensor is represented as the correlation $m n\left\langle u_{s}^{i} u_{s}^{j}\right\rangle$, which is exactly the term $\Phi_{s} R_{s}^{i j}$ in equation (16).

The conservation equation for $\frac{1}{2} \mathrm{~m} u_{s}^{2}$ matches the equation for granular tem- 
perature (19) if the flux, production and dissipation of fluctuating energy are respectively given by

$$
\begin{aligned}
& \Phi_{s} \mathcal{J}_{s}=\Phi_{s} \rho_{s}\left\langle\frac{1}{2} u_{s}^{2} u_{s}^{j}\right\rangle+\Theta_{s}^{j}\left(\frac{1}{2} \mathrm{~m} u_{s}^{2}\right) \\
& \mathcal{P}_{s}=-\left[\Phi_{s} \rho_{s}\left\langle u_{s}^{i} u_{s}^{j}\right\rangle+\Theta_{s}^{i}\left(\mathrm{~m} u_{s}^{j}\right)\right] \frac{\partial U_{s}^{i}}{\partial x^{j}} \\
& \Phi_{s} \epsilon_{s}=\chi_{s}\left(\frac{1}{2} \mathrm{~m} u_{s}^{2}\right)
\end{aligned}
$$

The form of the production term $\mathcal{P}_{s}$ in the dispersed phase deserves some comment. The production rate of fluctuating kinetic energy in the solid-phase is exclusively due to the kinetic and collisional stress tensors acting upon the mean velocity gradient. There is no production rate related to concentration gradients as suggested by the general form in (12). This is a direct result of the kinetic theory approach.

Closure relations are required for the above correlations in order to close the set of solid-phase transport equations. As it was mentioned earlier, expressions for the sink and flux terms of any particle property can be devised after solving their integral relations under some necessary assumptions [4]. It is assumed that the solid-phase flow consists of nearly elastic particles, $\left(1-e_{s}\right) \ll 1$, the spatial gradients of mean quantities are supposed to be small and low anisotropy of normal stresses ought to be expected.

Following the derivation of Peirano and Leckner [9], it is possible to express the kinetic and collisional stress tensors and the energy flux in terms of mean quantities and known flow parameters. The effect of an interstitial fluid on the particles motion is also take into account, which differs from the dry granular flow analysed by Jenkins and Richman [4]. However, both sets of constitutive relations match when there is no interstitial fluid.

Table 2 summarizes the set of equations for gas-solid flow under the Eulerian framework. Expressions for the solid-phase stress tensors, energy flux and collisional rate of dissipation are presented in the following sections together with constitutive relations and closures for the gas-phase, which allows to close the equation system. Now attention is directed to the interfacial coupling between phases.

\section{Closure modeling}

Explicit expressions for the momentum exchange and interfacial work terms are described in this section. Interaction terms represent the coupling between 
phases, so their forms need to be consistent with the physical phenomenon under consideration. Furthermore, closure relations for the stress tensors and energy flux on both phases are described. Finally, a $k$ - $\epsilon$ model for gas-particle flow is proposed based on the equations previously derived as well as the generally accepted ideas for turbulence modeling in single phase flow.

\subsection{Momentum exchange}

Gas-solid flows involve the transport of heavy particles $\left(\rho_{f} / \rho_{s} \sim 10^{-3}\right)$ being the drag force the most significant contribution to the momentum exchange term. Under the Eulerian framework, the instantaneous drag force acting on the gas-phase can be written as

$$
\tilde{m}_{f}^{i}=\tilde{\phi}_{s} \rho_{s} \tilde{\beta}\left(\tilde{u}_{s}^{i}-\hat{u}_{f}^{i}\right)
$$

where $\hat{u}_{f}^{i}$ is the instantaneous undisturbed fluid velocity and $\tilde{\beta}$ is a drag function which generally depends on the local properties of the carrier phase and turbulence, relative velocity, particle size and solid-phase concentration. This parameter, as expressed in Table 3, is the inverse of the particle response time, $\tau_{d}$. The undisturbed fluid velocity can be related to the actual carrier phase velocity by introducing the drifting velocity, $U_{d}^{i}$

$$
\hat{u}_{f}^{i}=\bar{U}_{f}^{i}+u_{f}^{i}+U_{d}^{i}
$$

The drifting velocity physically accounts for the dispersion of particles by the fluid turbulence. This velocity can be thought as to be the gas-phase fluctuating velocity seen by the particles at a specific point in the flow domain. According to Simonin [6], $U_{d}^{i}$ reduces to the single correlation between solidphase volume fraction fluctuation and gas-phase fluctuating velocity when the particle diameter and mean spacing values are tending to zero with respect to the smallest turbulent length scales. Based on a configuration of particles suspended in homogeneous turbulence, a model for the drifting velocity can be expressed as

$$
U_{d}^{i}=-\mathcal{D}_{s f}^{t}\left(\frac{1}{\Phi_{s}} \frac{\partial \Phi_{s}}{\partial x^{i}}-\frac{1}{\Phi_{f}} \frac{\partial \Phi_{f}}{\partial x^{i}}\right)
$$

where the turbulent dispersion coefficient, $\mathcal{D}_{s f}^{t}$, should apparently depend on the relative motion between a given particle and the surrounding fluid, or in other words, the so-called eddy-particle interaction time, $\tau_{s f}^{t}$. The extent to which a particle interacts with the surrounding fluid can be quantified by the Stokes number

$$
S t \equiv \frac{\text { Particle response time }}{\text { Characteristic flow time }}=\frac{\tau_{d}}{\tau_{f}^{t}}
$$

Fig. 1 illustrates how a particle trajectory can be affected by the fluid flow. As shown in the figure, any of three possible occurrences are likely to happen if a 


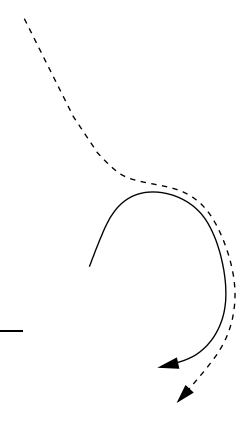

$S t \ll 1$

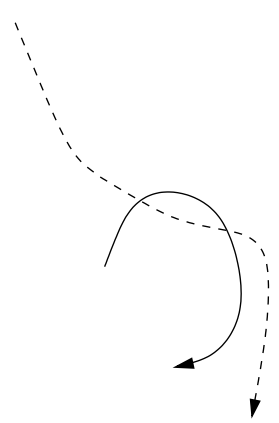

$S t \sim 1$

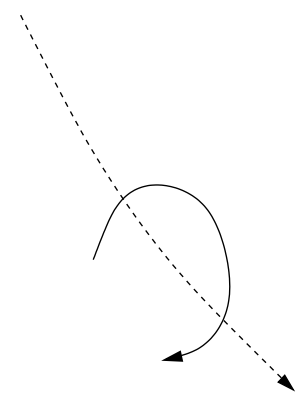

$S t \gg 1$

Fig. 1. Effect of an eddy (solid line) on particle trajectory (dashed line).

given particle encounters an eddy on its path through the continuous medium: the particle has sufficiently small inertia to precisely follow the eddy motion $(S t \ll 1)$, the eddy may cause a significant deviation on the particle trajectory $(S t \sim 1)$, or the particle is not affected by the eddy leaving the swirling flow with its path unaffected $(S t \gg 1)$. Hence, the trajectory followed by a particle depends on the particle inertia and the intensity of the coming eddy, both effects being considered by the Stokes number. Other relevant time scales in gas-solid flow are also listed in Table 3.

A simplification regarding the drag function is required before time-averaging the momentum transfer term (27). Following the assumptions described by Hrenya and Sinclair [16], fluctuations on the drag function are written as

$$
\tilde{\beta}=\bar{\beta}+\frac{\partial \tilde{\beta}}{\partial \tilde{\phi}_{s}} \phi_{s}+\frac{\partial \tilde{\beta}}{\partial \tilde{u}_{s}^{i}} u_{s}^{i}+\frac{\partial \tilde{\beta}}{\partial \tilde{u}_{f}^{i}} u_{f}^{i}+\text { H.O.T. }
$$

Considering a small particle size, contributions from fluctuations in (31) can be neglected. This is a reasonable assumption for dilute systems and it also implies that time scales associated with mean and fluctuating motions are assumed to be identical. Then, averaging (27) yields to

$$
\begin{aligned}
M_{f}^{i} & =\rho_{s} \bar{\beta}\left(\Phi_{s} U_{s}^{i}-\overline{\tilde{\phi}_{s} \hat{u}_{f}^{i}}\right) \\
& =\rho_{s} \bar{\beta}\left[\Phi_{s}\left(U_{s}^{i}-U_{f}^{i}-U_{d}^{i}\right)-\overline{\phi_{s} u_{f}}+\Phi_{s} \frac{\overline{\phi_{f} u_{f}}}{\Phi_{f}}\right]
\end{aligned}
$$

where $U_{s}^{i}$ and $U_{f}^{i}$ are Favre-averaged velocities (7). The second to last term on the right hand side, $\overline{\phi_{s} u_{f}}$, can be neglected if the solid-phase concentration fluctuation is uncorrelated to the gas-phase velocity field. Experimental studies have shown that heavy particles are not preferentially concentrated so their distribution should be uncorrelated with the velocity field [17]. This also implies that the last term can be disregarded. The mean velocities within 
Table 3

Representative time scales and flow parameters.

\begin{tabular}{|lcc|}
\hline $\bar{\beta}=\frac{1}{\tau_{d}}=\frac{\overline{C_{D}} R e_{p}}{24 \tau_{p}}$ & $\tau_{p}=\frac{\rho_{s} d_{s}^{2}}{18 \mu_{f}}$ & $R e_{p}=\frac{\rho_{f} \overline{\left|\tilde{\boldsymbol{u}}_{r}\right|} d_{s}}{\mu_{f}}$ \\
\hline$\tau_{f}^{t} \sim \frac{k_{f}}{\epsilon_{f}}$ & $\tau_{s f}^{t}=\tau_{f}^{t}\left(1+C_{\beta} \xi_{r}\right)^{-1 / 2}$ & $\xi_{r}=\frac{3\left|\boldsymbol{U}_{r}\right|^{2}}{2 k_{f}}$ \\
\hline$\tau_{s}^{c}=\frac{d_{s}}{24 \Phi_{s} g_{0}} \sqrt{\frac{\pi}{\Theta_{s}}}$ & $\eta=\frac{\tau_{s f}^{t}}{\tau_{d}}$ & $g_{0}=\left[1-\left(\frac{\Phi_{s}}{\Phi_{s, \text { max }}}\right)^{\frac{1}{3}}\right]^{-1}$ \\
\hline
\end{tabular}

brackets in (32) define the mean relative velocity

$$
U_{r}^{i}=U_{s}^{i}-U_{f}^{i}-U_{d}^{i}
$$

Finally, the mean drag force acting on the gas-phase can be written as

$$
M_{f}^{i}=\rho_{s} \bar{\beta} \Phi_{s} U_{r}^{i}
$$

The opposite force acting on the solid-phase, $M_{s}^{i}$, follows from equation (5).

The averaged drag function, $\bar{\beta}$, needed to determined the mean momentum coupling accounts for the deviation from the force acting on a single sphere (Stokes's law) [10]. This drag function can be recast in terms of particle Reynolds number, $R e_{p}$, a mean drag coefficient, $\overline{C_{D}}$, and the Stokesian time constant, $\tau_{p}$, as it is indicated in Table 3 . The drag coefficient suggested by Wen and $\mathrm{Yu}[1]$ is adopted in this study

$$
C_{D}=\left\{\begin{array}{lll}
\frac{24}{\Phi_{f} R e_{p}}\left[1+0.15\left(\Phi_{f} R e_{p}\right)^{0.687}\right] & \text { if } \quad \Phi_{f} R e_{p}<1000 \\
0.44 & \text { if } \quad \Phi_{f} R e_{p} \geq 1000
\end{array}\right.
$$

To agree with Wen and Yu's definition of drag function, it is required that $\overline{C_{D}}=C_{D}\left(1-\Phi_{s}\right)^{-1.65}$, which is also consistent with the relations given in Table 3. This corrects the drag coefficient showing the effect of a multiple number of particles in the flow. 


\subsection{Interfacial work}

The work done by the fluctuating drag force acting on each phase needs to be examined.

Regarding the gas-phase turbulent kinetic energy equation, interfacial energy exchange terms are obtained after substituting the instantaneous drag force (27) into the interfacial work term in equation (17). By considering the same assumptions required to derive the mean momentum exchange term, the interfacial work in the gas-phase turbulent kinetic energy equation can be expressed as

$$
\overline{u_{f}^{i} m_{f}^{i}}=\rho_{s} \bar{\beta}\left(\overline{\tilde{\phi}_{s} u_{f}^{i} \tilde{u}_{s}^{i}}-\overline{\tilde{\phi}_{s} u_{f}^{i} \hat{u}_{f}^{i}}\right)
$$

The above relation can be rewritten in terms of mean values using Reynolds decomposition and the definition of the undisturbed fluid velocity (28). It yields

$$
\begin{aligned}
\overline{u_{f}^{i} m_{f}^{i}}=\rho_{s} \bar{\beta}\left[\Phi_{s}\left(\overline{u_{s}^{i} u_{f}^{i}}-\overline{u_{f}^{i} u_{f}^{i}}\right)+\overline{\phi_{s} u_{s}^{i} u_{f}^{i}}\right. & -\overline{\phi_{s} u_{f}^{i} u_{f}^{i}}+ \\
& \left.\left(\bar{U}_{s}^{i}-\bar{U}_{f}^{i}-U_{d}^{i}\right) \overline{\phi_{s} u_{f}^{i}}\right]
\end{aligned}
$$

For particles with relatively large Stokes numbers $(S t>1)$, the contribution from the last three terms in (37) is negligible as pointed out in [17] and [6]. In terms of modeled correlations, the coupling energy term in the gas-phase turbulent kinetic energy equation (17) becomes

$$
\overline{u_{f}^{i} m_{f}^{i}}=\rho_{s} \Phi_{s} \bar{\beta}\left(k_{s f}-2 k_{f}\right)
$$

These terms respectively represent mean rate of interfacial energy transfer as a result of the correlation between velocity fluctuations of the carrier and dispersed phases, $k_{s f}$, and extra-dissipation owing to the fluctuating drag force acting on the fluid elements.

Similarly, the interfacial work in the solid-phase fluctuating kinetic energy equation (19) becomes

$$
\overline{u_{s}^{i} m_{s}^{i}}=\rho_{s} \bar{\beta}\left(\overline{\tilde{\phi}_{s} u_{s}^{i} \hat{u}_{f}^{i}}-\overline{\tilde{\phi}_{s} u_{s}^{i} \tilde{u}_{s}^{i}}\right)
$$

which can be rewritten as

$$
\begin{aligned}
\overline{u_{s}^{i} m_{s}^{i}}=\rho_{s} \bar{\beta}\left[\Phi_{s}\left(\overline{u_{s}^{i} u_{f}^{i}}-\overline{u_{s}^{i} u_{s}^{i}}\right)+\overline{\phi_{s} u_{s}^{i} u_{f}^{i}}\right. & -\overline{\phi_{s} u_{s}^{i} u_{s}^{i}}+ \\
& \left.-\left(\bar{U}_{s}^{i}-\bar{U}_{f}^{i}-U_{d}^{i}\right) \overline{\phi_{s} u_{s}^{i}}\right]
\end{aligned}
$$


Table 4

Models for gas-solid velocity correlation.

\begin{tabular}{|c||c|}
\hline Model 1: Isotropic model & Model 3: Simonin model [6] \\
\hline$k_{s f}=c_{s f} \sqrt{k_{f} \Theta_{s}}$ & \multirow{2}{*}{$k_{s f}=\frac{\eta}{1+\eta\left(1+\mathcal{X}_{s f}\right)}\left(2 k_{f}+3 \mathcal{X}_{s f} \Theta_{s}\right)$} \\
\cline { 1 - 1 }$k_{s f}=\frac{\bar{\beta} d_{s}}{4 \sqrt{\pi \Theta_{s}}}\left|\boldsymbol{U}_{r}\right|^{2}$ & where $\quad \mathcal{X}_{s f}=\frac{\Phi_{s} \rho_{s}}{\Phi_{f} \rho_{f}}$ \\
\hline
\end{tabular}

By regarding the assumptions previously stated, the last three terms in (40) can be neglected. In terms of modeled correlations, the coupling energy term in the granular temperature equation becomes

$$
\overline{u_{s}^{i} m_{s}^{i}}=\rho_{s} \Phi_{s} \bar{\beta}\left(k_{s f}-3 \Theta_{s}\right)
$$

where the second term on the right hand side stands for the extra-dissipation due to the work done by the fluctuating drag force acting upon the particles in fluctuating motion.

There is no general agreement on how to model neither the fluid-solid fluctuating velocity correlation, $k_{s f}$, nor the drifting velocity. A relatively simple closure model for $k_{s f}$ can be obtained by assuming isotropic velocity fluctuations within each phase. A similar model was considered to simulate turbulent gas-solid flow in a vertical pipe [2]. Good agreement was found between the simulation results and experimental data for mean and fluctuating velocities on both phases. However, the isotropic model contribution in the carrier phase turbulent kinetic energy equation seems to overpredict the total production of $k_{f}$, leading to a high turbulence intensity across the pipe.

Koch [18] was among the first to develop a model for the fluctuating fluidsolid velocity correlation, which may be valid for a dilute gas-solid suspension at low particle Reynolds numbers in the limit where particle-particle collisions determine the particle velocity distribution function. On the other hand, closure assumptions regarding $k_{s f}$ must be consistent with the modeling of single-phase turbulence when the particle response time tends to zero with respect to the eddy-particle interaction time (i.e. $\eta \rightarrow \infty$ ), as pointed out by Simonin [6].

More detailed models for the fluid-solid velocity correlation can be proposed by developing modeled transport equations for $k_{s f}$ and $U_{d}^{i}[9]$. However, in order to have a consistent level of closure only algebraic relations are considered. 
Based on the current models for $k_{s f}$, the performance of three different closure models is analyzed in this study. The assessed models are listed in table 4 . The turbulent dispersion coefficient, $\mathcal{D}_{s f}^{t}$, necessary to determine the drifting velocity, can be related to the fluid-solid velocity correlation as

$$
\mathcal{D}_{s f}^{t}=\frac{1}{3} \tau_{s f}^{t} k_{s f}
$$

As there exists a relative velocity between a particle and the surrounding gas, a moving particle will cross the trajectories of fluid particles. Therefore, the gas-phase turbulent motion must be expressed as viewed by the particulate phase, being $\tau_{s f}^{t}$ the time scale of the fluid turbulent motion determined along particle trajectories [6]. The direction-dependent parameter, $C_{\beta}$, needed to compute the eddy-particle interaction time which is listed in Table 3, is given by

$$
C_{\beta}=1.8-1.35\left[\frac{U_{r}^{i} U_{s}^{i}}{\left|\boldsymbol{U}_{r}\right|\left|\boldsymbol{U}_{s}\right|}\right]^{2}
$$

And the magnitude of the instantaneous relative velocity necessary to calculate the particle Reynolds number and mean drag function can be approximated as

$$
\overline{\left|\tilde{\boldsymbol{u}}_{r}\right|} \approx \sqrt{U_{r}^{i} U_{r}^{i}+\overline{u_{r}^{i} u_{r}^{i}}}=\sqrt{U_{r}^{i} U_{r}^{i}+\frac{2}{3}\left(k_{f}-k_{s f}\right)+\Theta_{s}}
$$

\subsection{Constitutive relations}

The equation set in Table 2 describes the evolution of mean velocities, pressure, turbulence kinetic energy, granular temperature and solid-phase concentration. This system, however, is not closed as the averaged transport equations contain several expressions that need to be related to mean variables. Namely, constitutive relations are required for the stress tensors and energy flux relative to each phase.

\subsubsection{Dispersed phase}

The Boussinesq assumption is used to model the solid-phase kinetic stress tensor

$$
R_{s}^{i j}=2 \rho_{s} \nu_{s}^{t}\left(S_{s}^{i j}-\frac{1}{3} S_{s}^{k k} \delta^{i j}\right)-\rho_{s} \Theta_{s} \delta^{i j}
$$

where $\nu_{s}^{t}$ stands for the solid-phase kinetic viscosity. Similarly, the collisional stress tensor is

$$
\mathcal{T}_{s}^{i j}=2 \rho_{s} \nu_{s}^{c}\left(S_{s}^{i j}-\frac{1}{3} S_{s}^{k k} \delta^{i j}\right)-\left(1+e_{s}\right)\left(2 \Phi_{s} \rho_{s} g_{0} \Theta_{s}-\mu_{f} \frac{\tau_{p}}{\tau_{s}^{c}} S_{s}^{k k}\right) \delta^{i j}
$$

where $\nu_{s}^{c}$ is the solid-phase collisional viscosity. Expressions for the solid-phase viscosities, given by kinetic theory, are listed in Table 5 . 
Table 5

Solid-phase viscosities and diffusion coefficients from kinetic theory [9].

\begin{tabular}{|c|c|}
\hline \multicolumn{2}{|l|}{ Stress tensor } \\
\hline$\nu_{s}^{t}=\left[\frac{2}{3} \eta k_{s f}+\Theta_{s}\left(1+\Phi_{s} g_{0} a_{1}\right)\right]\left(\frac{2}{\tau_{d}}+\frac{a_{2}}{\tau_{s}^{c}}\right)^{-1}$ & $a_{1}=\frac{2}{5}\left(1+e_{s}\right)\left(3 e_{s}-1\right)$ \\
\hline$\nu_{s}^{c}=\frac{4}{5} \Phi_{s} g_{0}\left(1+e_{s}\right)\left(\nu_{s}^{t}+d_{s} \sqrt{\frac{\Theta_{s}}{\pi}}\right)$ & $a_{2}=\frac{1}{5}\left(1+e_{s}\right)\left(3-e_{s}\right)$ \\
\hline \multicolumn{2}{|l|}{ Energy flux } \\
\hline$\kappa_{s}^{t}=\left[\frac{3}{5} \eta k_{s f}+\Theta_{s}\left(1+\Phi_{s} g_{0} a_{3}\right)\right]\left(\frac{9}{5 \tau_{d}}+\frac{a_{4}}{\tau_{s}^{c}}\right)^{-1}$ & $a_{3}=\frac{3}{5}\left(1+e_{s}\right)^{2}\left(2 e_{s}-1\right)$ \\
\hline$\kappa_{s}^{c}=\Phi_{s} g_{0}\left(1+e_{s}\right)\left(\frac{6}{5} \kappa_{s}^{t}+\frac{4}{3} d_{s} \sqrt{\frac{\Theta_{s}}{\pi}}\right)$ & $a_{4}=\frac{1}{100}\left(1+e_{s}\right)\left(49-33 e_{s}\right)$ \\
\hline
\end{tabular}

Energy flux is modeled as proportional to the granular temperature gradient

$$
\mathcal{J}_{s}^{j}=-\Gamma_{s} \frac{\partial \Theta_{s}}{\partial x^{j}}
$$

where $\Gamma_{s}=\frac{3}{2} \rho_{s}\left(\kappa_{s}^{t}+\kappa_{s}^{c}\right)$ is the granular conductivity which consists of kinetic $\left(\kappa_{s}^{t}\right)$ and collisional $\left(\kappa_{s}^{c}\right)$ contributions. Solid-phase conductivities are given in Table 5 .

The collisional rate of dissipation can be written as

$$
\epsilon_{s}=\rho_{s} \frac{\left(1-e_{s}^{2}\right)}{2 \tau_{s}^{c}} \Theta_{s}
$$

where the particle-particle collision time, $\tau_{s}^{c}$, accounts for the time experienced by a given particle between two successive binary collisions.

\subsubsection{Carrier phase}

For a Newtonian fluid, the mean viscous stress tensor is given by

$$
\mathcal{T}_{f}^{i j}=2 \mu_{f}\left(S_{f}^{i j}-\frac{1}{3} S_{f}^{k k} \delta^{i j}\right)
$$


In two-equation turbulence modeling, Boussinesq assumption is commonly employed to determine the Reynolds stresses

$$
R_{f}^{i j}=2 \mu_{f}^{t}\left(S_{f}^{i j}-\frac{1}{3} S_{f}^{k k} \delta^{i j}\right)-\frac{2}{3} \rho_{f} k_{f} \delta^{i j}
$$

where $R_{f}^{i j}$ is aligned with the mean rate-of-strain tensor. This alignment is not always the case for most turbulent flows and it represents a significant potential discrepancy [19]. Furthermore, Boussinesq assumption implies that Reynolds stresses will only be present if there is a velocity gradient, which does not contemplate velocity disturbances generated by the dispersed phase.

The energy flux is modeled by a gradient-diffusion relation

$$
\mathcal{J}_{f}^{j}=-\left(\mu_{f}+\frac{\mu_{f}^{t}}{\sigma_{k}}\right) \frac{\partial k_{f}}{\partial x^{j}}
$$

\section{Eddy-viscosity}

The eddy-viscosity, $\mu_{f}^{t}$, in (50) and (51) may be determined from local values of two scalar properties that characterize the gas turbulence, i.e. a turbulent velocity scale, $\mathcal{U}$, and a turbulent length scale, $\ell$

$$
\frac{\mu_{f}^{t}}{\rho_{f}} \sim \mathcal{U} \ell
$$

It is customary to estimate the velocity scale as $\mathcal{U}=k_{f}^{1 / 2}$. The length scale which quantifies the turbulent transport or mixing process can be determined by combining $k_{f}$ and $\epsilon_{f}$. However, the effect of particles on the turbulent length scale should also be taken into account.

As the space occupied by the spherical particles is not available to the gas, this must restrict the turbulent fluctuations. The space occupied by the particles in a volume $\ell^{3}$ is given by

$$
\Phi_{s} \ell^{3}=\frac{\pi}{6} d_{s}^{3} N
$$

where $N$ is the number of particles in that volume. Furthermore, an assumption must be made for the structure, or arrangement, of the particles. If it is assumed that particles are evenly distributed along the three spatial directions, then

$$
\ell^{\prime}=\ell-N^{1 / 3} d_{s}=\ell\left[1-\left(\frac{6 \Phi_{s}}{\pi}\right)^{1 / 3}\right]
$$

Nevertheless, the gas-phase turbulence will be suppressed at the close-packing limit, so the space occupied by the particles should be normalized by the maximum solids volume fraction [20]. Thus, the effective turbulent length 
Table 6

$k-\epsilon$ turbulence model coefficients.

\begin{tabular}{|c|c|c|c|c|c|}
\hline$c_{\mu}$ & $c_{\epsilon 1}$ & $c_{\epsilon 2}$ & $c_{\epsilon 3}$ & $\sigma_{k}$ & $\sigma_{\epsilon}$ \\
\hline 0.09 & 1.44 & 1.92 & 1.2 & 1.0 & 1.3 \\
\hline
\end{tabular}

scale becomes

$$
\ell_{e}=\ell\left[1-\left(\frac{6 \Phi_{s}}{\pi \Phi_{s, \max }}\right)^{1 / 3}\right] \sim \frac{k_{f}^{3 / 2}}{\epsilon_{f}}
$$

where the maximum packing fraction is to be determined experimentally. Hence, the eddy viscosity can be expressed as

$$
\mu_{f}^{t}=c_{\mu} \rho_{f}\left[1-\left(\frac{6 \Phi_{s}}{\pi \Phi_{s, \max }}\right)^{1 / 3}\right]^{-1} \frac{k_{f}^{2}}{\epsilon_{f}}
$$

\section{$4.4 k-\epsilon$ model for gas-solid flow}

A modeled transport equation for the gas-phase turbulent kinetic energy finally takes the form

$$
\begin{aligned}
\frac{\partial}{\partial t}\left(\rho_{f} \Phi_{f} k_{f}\right)+\frac{\partial}{\partial x^{i}}\left(\rho_{f} \Phi_{f} U_{f}^{i} k_{f}\right)= & \frac{\partial}{\partial x^{j}}\left[\Phi_{f}\left(\mu_{f}+\frac{\mu_{f}^{t}}{\sigma_{k}}\right) \frac{\partial k_{f}}{\partial x^{j}}\right]+ \\
& \mathcal{P}_{f}-\Phi_{f} \epsilon_{f}+\rho_{s} \Phi_{s} \bar{\beta}\left(k_{s f}-2 k_{f}\right)
\end{aligned}
$$

where production due to mean flow gradients, $\mathcal{P}_{f}$, needs no further modeling once the Reynolds stress, $R_{f}^{i j}$, has been approximated by the Bousinessq or eddy-viscosity model (50). The gas-phase turbulence modulation due to gassolid interaction and extra-dissipation (38) are already expressed in terms of mean flow quantities.

Similarly, an analogous modeled transport equation for the gas-phase rate of dissipation, $\epsilon_{f}$, can be written as

$$
\begin{array}{r}
\frac{\partial}{\partial t}\left(\rho_{f} \Phi_{f} \epsilon_{f}\right)+\frac{\partial}{\partial x^{i}}\left(\rho_{f} \Phi_{f} U_{f}^{i} \epsilon_{f}\right)=\frac{\partial}{\partial x^{j}}\left[\Phi_{f}\left(\mu_{f}+\frac{\mu_{f}^{t}}{\sigma_{\epsilon}}\right) \frac{\partial \epsilon_{f}}{\partial x^{j}}\right]+ \\
\left(c_{\epsilon 1} \mathcal{P}_{f}-c_{\epsilon 2} \Phi_{f} \epsilon_{f}\right) \frac{\epsilon_{f}}{k_{f}}+c_{\epsilon 3} \rho_{s} \Phi_{s} \bar{\beta}\left(k_{s f}-2 k_{f}\right) \frac{\epsilon_{f}}{k_{f}}
\end{array}
$$

Suitable model coefficients are listed in Table 6 . When the gas-solid velocity correlation is modeled by using Model 1 in Table 4 , the coefficient $c_{s f}$ is set to 0.2 . 


\subsection{Solid-phase fluctuating kinetic energy}

The granular temperature transport equation takes the following form

$$
\begin{aligned}
\frac{3}{2}\left[\frac{\partial}{\partial t}\left(\rho_{s} \Phi_{s} \Theta_{s}\right)+\frac{\partial}{\partial x^{i}}\left(\rho_{s} \Phi_{s} U_{s}^{i} \Theta_{s}\right)\right]= & \frac{\partial}{\partial x^{j}}\left[\Phi_{s} \Gamma_{s} \frac{\partial \Theta_{s}}{\partial x^{j}}\right]+\mathcal{P}_{s}-\Phi_{s} \epsilon_{s} \\
& +\rho_{s} \Phi_{s} \bar{\beta}\left(k_{s f}-3 \Theta_{s}\right)
\end{aligned}
$$

where the production term, $\mathcal{P}_{s}$, is given by

$$
\mathcal{P}_{s}=\Phi_{s}\left(R_{s}^{i j}+\mathcal{T}_{s}^{i j}\right) \frac{\partial U_{s}^{i}}{\partial x^{j}}
$$

\section{Model validation}

Simplified versions of the transport equations listed in Table 2 are applied to simulate a steady, fully-developed flow in a vertical pipe of $30.5 \mathrm{~mm}$ diameter. This case was experimentally researched by Tsuji et al. [12]. Fig. 2 shows a schematic of the flow region together with relevant variables and parameters. A constant pressure gradient in the axial direction, $\frac{d P}{d z}$, is set as the flow driving force acting against gravity. Thus, pressure gradient is an input parameter which needs to be adjusted in order to attain a specific flow condition.

In addition, three different models accounting for the gas-solid velocity correlation are employed to determine the coupling between gas and solid fluctuating kinetic energy equations and the effect of gas-phase turbulence on the solid-phase constitutive relations.

Predicted mean velocities and turbulence intensity profiles are compared with a single set of measurements of Tsuji et al. [12]. The representative case chosen for validation comprises polystyrene beads with density $\rho_{s}=1020 \mathrm{~kg} / \mathrm{m}^{3}$ and diameter $d_{s}=200 \mu \mathrm{m}$. A mass-loading ratio, $\mathcal{L}$, of 3.2 which is defined as

$$
\mathcal{L}=\frac{\int_{0}^{R} \rho_{s} \Phi_{s} U_{s} r d r}{\int_{0}^{R} \rho_{f}\left(1-\Phi_{s}\right) U_{f} r d r}
$$

and a centerline gas velocity $U_{c l}=10.8 \mathrm{~m} / \mathrm{s}$ are also reported in these experiments.

For a steady, fully-developed and relatively dilute gas-solid flow in a vertical pipe, the gas-solid flow transport equations can be simplified to a set of non- 


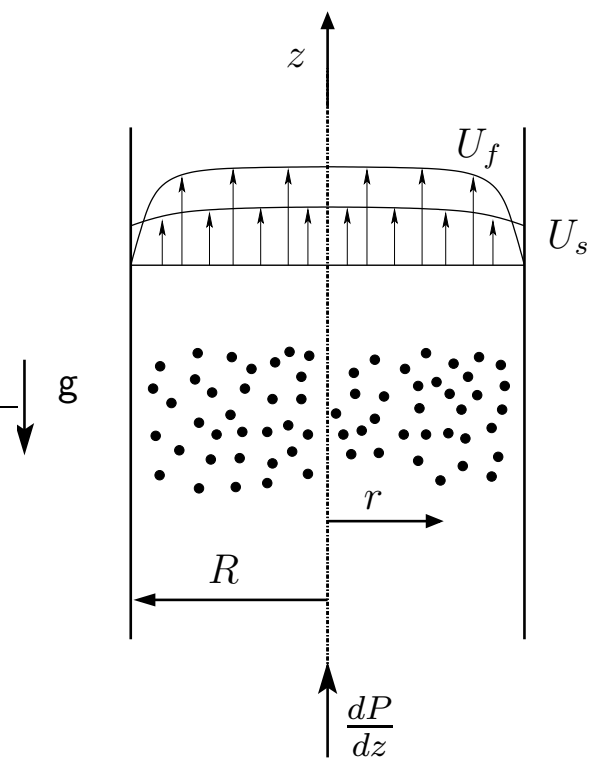

Fig. 2. Gas-solid flow in a vertical pipe of radius $R$. $U_{f}$ and $U_{s}$ stand for the gas and solid phase mean velocities, respectively.

linear ordinary differential equations (in polar coordinates) for determining the six unknowns $U_{f}, U_{s}, \Phi_{s}, \Theta_{s}, k_{f}, \epsilon_{f}$. The equation system is summarized in Table 7 . The gas-phase equations include balance of momentum in the axial direction, turbulent kinetic energy and rate of dissipation. On the other hand, momentum equations in the axial and radial directions need to be satisfied for the solid-phase together with the granular temperature equation. It can be shown by looking at equations 16, 45 and 46 that the solid-phase momentum equation in the radial direction simplifies to $0=\frac{d P_{s}}{d r}$, where $P_{s}$ is the so-called granular pressure. Besides, the solid-phase shear viscosity is defined as $\mu_{s}=\Phi_{s} \rho_{s}\left(\nu_{s}^{t}+\nu_{s}^{c}\right)$ and the rate of collisional energy dissipation (48) is recast as a linear term on $\Theta_{s}$ by defining

$$
\lambda_{s}=\Phi_{s} \rho_{s} \frac{\left(1-e_{s}^{2}\right)}{2 \tau_{s}^{c}}
$$

As it was previously stated in section 3.1, a very important and sensitive parameter that characterizes particle-particle interaction is the coefficient of restitution. As a result, it will significantly influence the calculation of solidphase constitutive relations like viscosities, diffusivities and collisional dissipation. It is not easy to specify a unique value for this coefficient as it depends on the impact velocity. Based on the assumptions of the current closure modeling, a constant value of $e_{s}$ may be adopted to account for the inelasticity of binary collisions. By conducting a survey of previous numerical studies (Hadinoto et al. [2], Zhang et al. [20], Cao et al. [21]) a value of 0.9 seems to be reasonable. This value is set constant throughout the simulations. 
Table 7

Simplified transport equations for fully-developed gas-solid flow in a vertical pipe

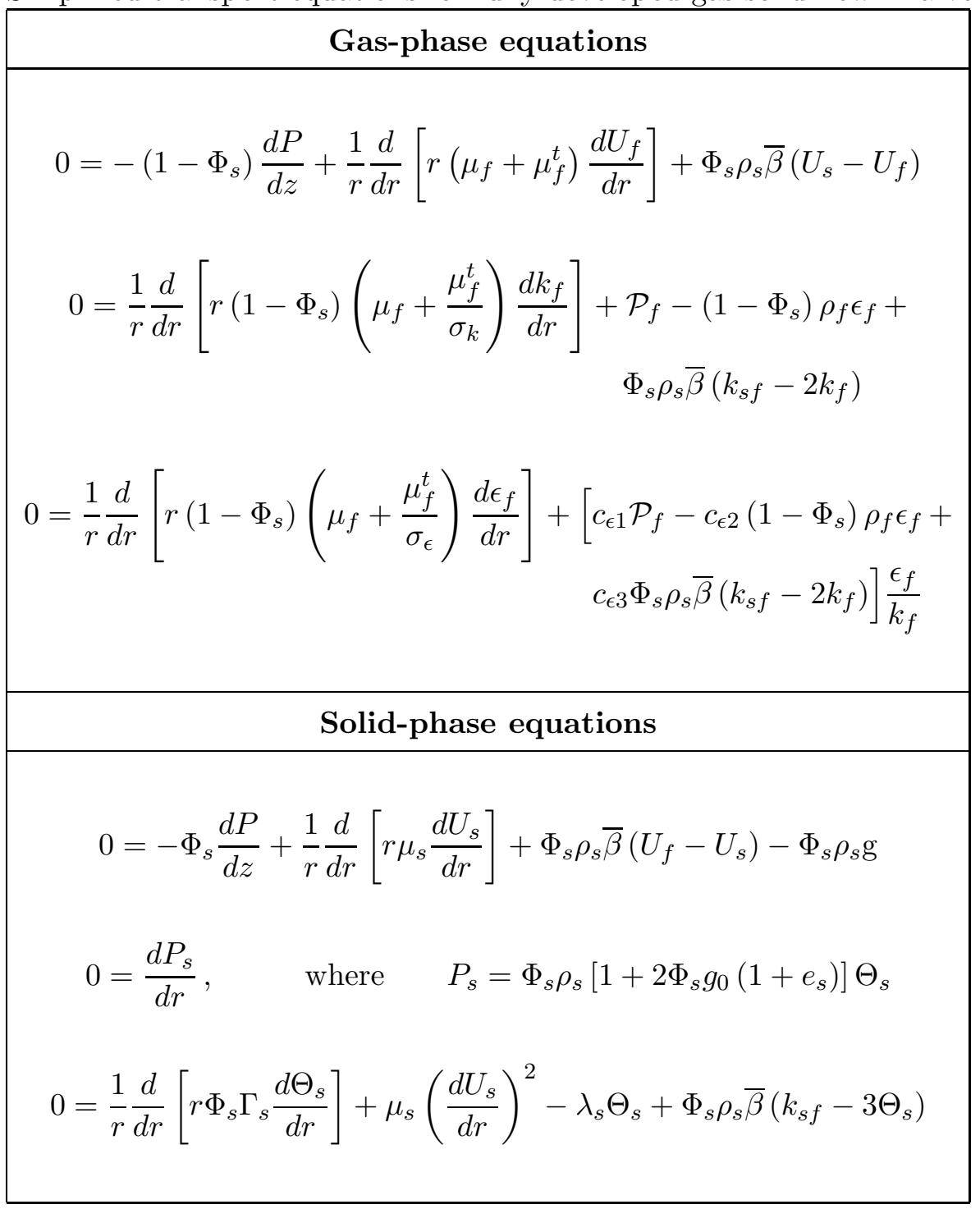

\subsection{Boundary conditions}

Standard single-phase wall boundary conditions for the gas-phase are assumed to hold in the case of dilute gas-solid flow

$$
\left.U_{f}\right|_{\text {wall }}=0,\left.\quad k_{f}\right|_{\text {wall }}=0,\left.\quad \epsilon_{f}\right|_{\text {wall }}=\frac{c_{\mu}^{3 / 4} k_{f, p}^{3 / 2}}{\kappa \delta r_{p}}
$$

where $\kappa=0.41$ and $\delta r$ is the distance from the wall to the nearest node $p$. This set of wall boundary conditions implies that the gas-phase mean velocity profile is not significantly affected by the presence of particles in the vicinity of the wall. 
Solid-phase boundary conditions at the pipe wall are slightly more complicated to specify as many rheological parameters need to be regarded. Particle linear and rotational velocity prior to collision, particle impact angle, particle shape and wall roughness are among the major parameters which are necessary for a complete characterization of particle-wall collisions [22]. On the other hand, more simplified wall boundary conditions were proposed by Johnson and Jackson [23] and are widely used for gas-solid flow. These are adopted in the present study in order to cope with a reasonable level of closure.

Similar to the use of wall functions in single-phase turbulent flow, a solid-phase shear stress is prescribed at node $p$

$$
\tau_{s}=\frac{\pi \Phi_{s, p}}{6 \Phi_{s, \max }} \varphi \rho_{s} g_{o} U_{s, p} \sqrt{3 \Theta_{s, p}}
$$

where the specularity coefficient, $\varphi$, represents the fraction of collisions that transfer momentum to the wall. This parameter varies from 0 to 1 for smooth to rough surfaces, respectively.

Similarly, the boundary condition for the granular temperature is based on a balance of energy in the vicinity of the pipe wall

$$
\left.\Gamma_{s} \frac{d \Theta_{s}}{d r}\right|_{\text {wall }}=U_{s, p} \tau_{s}-I_{s}
$$

$$
\text { where } \quad I_{s}=\frac{\pi \Phi_{s, p}}{12 \Phi_{s, \max }} \rho_{s} g_{o}\left(1-e_{w}^{2}\right)\left(3 \Theta_{s, p}\right)^{3 / 2}
$$

where $e_{w}$ is the coefficient of restitution for collisions between particles and the pipe wall. Thus, the flux of granular temperature at the wall is determined from a balance between generation of granular energy due to particle slip at the wall and dissipation due to inelastic collisions, $I_{s}$.

The above wall boundary conditions for the solid-phase velocity and granular temperature have shown to be fairly stable. On the other hand, setting the gradients of $U_{s}$ and $\Theta_{s}$ to zero leads to oscillations and divergence. Optimal values for the particle-wall collision and specularity coefficients are 0.85 and 0.005 , respectively. The granular temperature profile shows some sensitivity to the experimental parameters $e_{w}$ and $\varphi$ which both depend on the properties of the particle and wall surface.

Due to the axisymmetrical nature of the flow, gradients of mean quantities are set to zero at the centerline, $\frac{d F}{d r}=0$. 


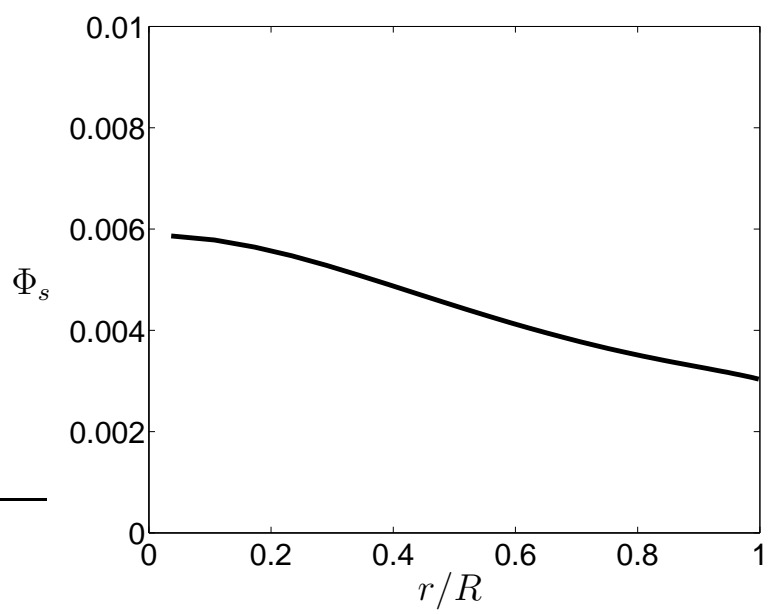

Fig. 3. Solid-phase volume fraction profile for a mass-loading ration $\mathcal{L}=3.2$.

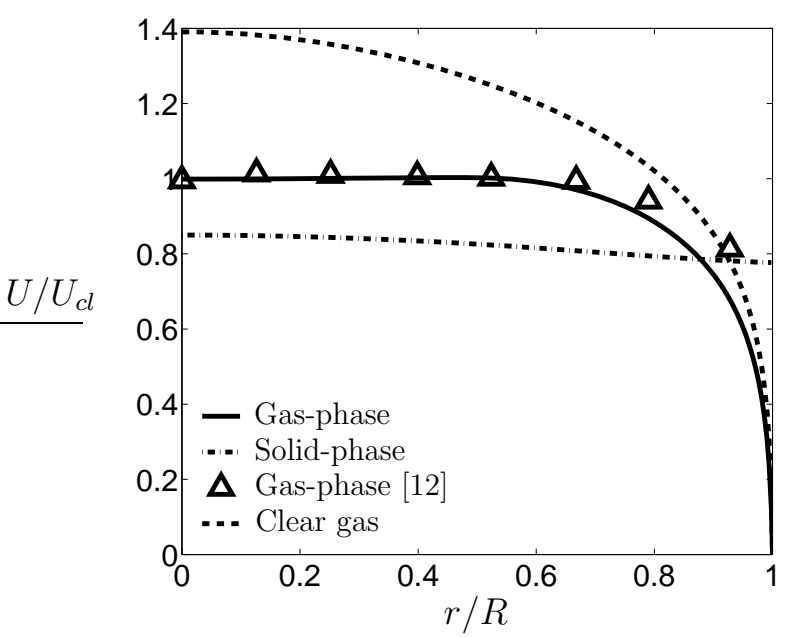

Fig. 4. Mean velocity profiles normalized by the centerline velocity, $U_{c l}=10.8 \mathrm{~m} / \mathrm{s}$.

\subsection{Numerical results}

The resulting equations are discretized by using the finite-volume method approach described in [24]. The need of low under-relaxation factors for mean flow quantities and source terms is necessary in order to achieve convergence. In the calculation, a constant pressure gradient is specified and tuned until the centerline velocity and mass-loading ratio are within $1 \%$ of the reported experimental values. In addition, three different models accounting for the gas-solid velocity correlation are employed to determine the coupling between gas and solid fluctuating kinetic energy equations and the effect of gas-phase turbulence on the solid-phase constitutive relations. The solution has been verified to be grid independent by checking that an increase in the number of grid points had a negligible effect on the computed profiles. 


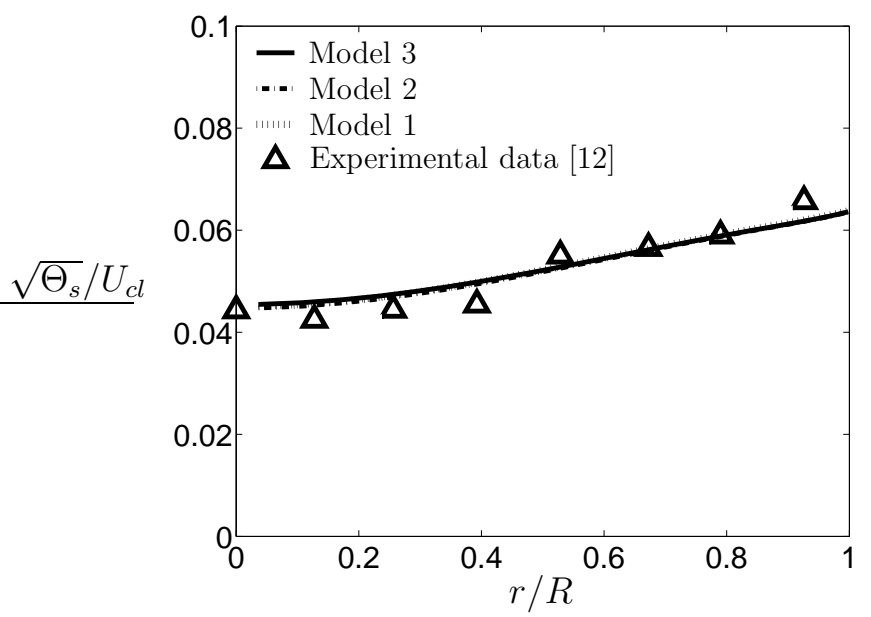

Fig. 5. Normalized granular temperature profiles for different models of gas-solid velocity correlation listed in Table 4.

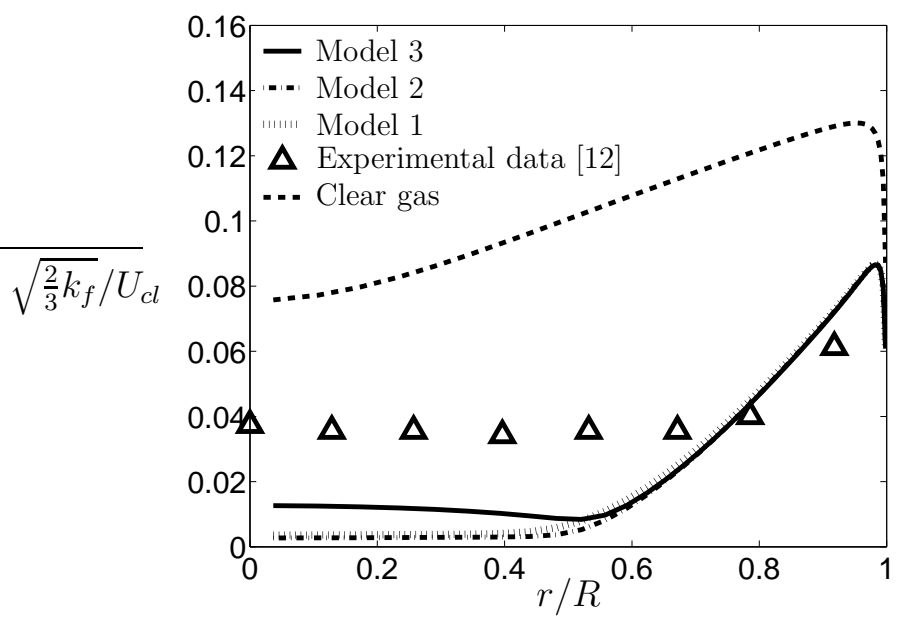

Fig. 6. Normalized gas-phase turbulence intensity profiles for different models of gas-solid velocity correlation listed in Table 4.

Fig. 3 illustrates the computed solid-phase concentration. It evidences a considerable segregation of particles towards the centerline. This is in accordance with previous numerical simulations [21] that show how particles move from high turbulence intensity regions to low turbulence regions.

Calculated gas and solid mean velocity profiles are shown in Fig. 4 together with available experimental measurements (only for air). The velocity profile for unloaded air flow is also shown to indicate the effect of the particulate phase on the gas phase mean velocity. There is very good agreement between the simulation results and the reported experimental data. The momentum exchange term is mainly responsible for the flattening of the gas mean velocity profile with respect to the clear gas. Although there are no solid-phase mean velocity measurements for this particular mass-loading ration, flat velocity 
profiles have also been observed for different particle loadings [21]. Unfortunately there is no complete set of experimental data for a single $\mathcal{L}$.

The dimensionless profile of granular temperature is shown in Fig. 5. There is excellent agreement between the computed profile and the solid-phase r.m.s. fluctuating velocity measurements by Tsuji et al. [12]. As it can be seen, granular temperature profiles are independent of the model employed to calculate the gas-solid velocity correlation.

Finally, normalized gas-phase turbulence intensity profiles are shown in Fig. 6 for different models of $k_{s f}$. It can be seen that there is an obvious turbulence attenuation with respect to the clear gas turbulence intensity profile. There is no apparent effect of $k_{s f}$ on turbulence intensity profiles in the vicinity of the pipe wall. This is expected as the production term due to the gas-phase mean velocity gradient dominates in the near wall region. However, the influence of $k_{s f}$ becomes important far from the pipe wall. It can also be related to the higher solid-phase concentration towards the centerline.

Although models 1 and 2 for $k_{s f}$ are quite different, their influence on turbulence intensity is almost the same. Overall, $\sqrt{\frac{2}{3} k_{f}} / U_{c l}$ is underestimated far from the wall independently of the chosen $k_{s f}$ model. Nevertheless, model 3 slightly improves the predicted turbulence intensity profile although its relative contribution is still too low.

\section{Conclusions}

This paper presents a general framework for the derivation of the averaged transport equations for turbulent gas-solid flows, taking into account the coupling between the turbulent nature of the gas phase and its interaction with the particles. It is shown that the interaction terms arise from the decomposed and Favre averaged Eulerian-Eulerian governing equations. To close the transport equations describing the particulate phase, the particulate stress tensor and energy flux are related to mean flow quantities by extending kinetic theory of granular flow with interstitial fluid effects. The solid phase constitutive equations are extended to include the effect of interstitial gas, momentum and energy exchange between phases, and turbulence modulation.

The model is validated by comparing its predictions to experiments of fully developed turbulent gas-solid pipe flow. In general, there is very good qualitative and quantitative agreement between the simulation results and the experimental data. The presence of a dispersed phase clearly modifies the gasphase behavior as illustrated when comparing the profiles for a single phase flow (clear gas) to the particle laden flow cases. It has also been shown that an 
increase in the solid-phase concentration significantly dampens the gas phase velocity fluctuations far from the pipe wall; this is in accordance with experimental observations. The gas fluctuating velocity is somewhat underestimated far from the pipe wall, which is probably due to the isotropic character of the model employed to determine the fluctuations on each phase. A more complete model, accounting for the anisotropy of the fluctuations, will improve the results even further.

\section{Acknowledgements}

This work has been financially supported by STEM (Energimyndigheten), the Swedish Energy Agency. This support is gratefully acknowledged.

\section{References}

[1] B. van Wachem, Derivation, implementation and validation of computer simulation models for gas-solid fluidized beds, Dissertation, Delft University of Technology, Delft, The Netherlands (2000).

[2] K. Hadinoto, J. Curtis, Effect of interstitial fluid on particle-particle interactions in kinetic theory approach of dilute turbulent fluid-particle flow, I $\&$ EC Research 43 (2004) 3604-3615.

[3] C. Lun, S. Savage, D. Jeffrey, N. Chepurniy, Kinetic theories for granular flow: inelastic particles in Couette flow and slightly inelastic particles in a general flowfield, J. Fluid Mech. 140 (1984) 223-256.

[4] J. Jenkins, M. Richman, Grad's 13-Moment System for a Dense Gas of Inelastic Spheres, Arch. Ration Mech. Anal. 87 (1985) 355-377.

[5] M. Reeks, On a kinetic equation for the transport of particles in turbulent flows, Physics of Fluids A 3 (1991) 446-456.

[6] O. Simonin, Continuum modelling of dispersed turbulent two-phase flows, Tech. Rep. Lecture Series 1996-02, Laboratoire National d'Hydraulique - EDF 6 Quai Watier - 78400 Chatou/France. (1996).

[7] Y. Zhang, J. Reese, Particle-gas turbulence interactions in a kinetic theory approach to granular flows, International Journal of Multiphase Flow 27 (2001) $1945-1964$.

[8] C. Lun, S. Savage, Kinetic Theory for Inertia Flows of Dilute Turbulent Gas-Solids Mixtures. In Lecture Notes in Physics: Theory of Granular Gases, Springer, New York, 2003. 
[9] E. Peirano, B. Leckner, Fundamentals of turbulent gas-solid flows applied to circulating fluidized bed combustion, Prog. Energy Combust. Sci. 24 (1998) 259-296.

[10] C. Crowe, M. Sommerfeld, Y. Tsuji, Multiphase Flows with Droplets and Particles, CRC Press LLC, 1998.

[11] D. Haworth, S. Pope, A generalized Langevin model for turbulent flows, Physics of Fluids 29 (1986) 387-405.

[12] Y. Tsuji, Y. Morikawa, H. Shiomi, LDV measurements of an air-solid two-phase flow in a vertical pipe, J. Fluid Mech. 139 (1984) 417-434.

[13] D. Drew, S. Passman, Theory of Multicomponent Fluids. Applied Mathematical Sciences 135, Springer, New York, 1999.

[14] S. Elgobashi, T. Abou-Arab, A two-equation turbulence model for two-phase flows, Physics of Fluids 26 (4) (1983) 931-938.

[15] S. Chapman, T. Cowling, The Mathematical Theory of Non-uniform Gases, Cambridge University Press, Cambridge, U.K., 1970.

[16] C. Hrenya, J. Sinclair, Effects of Particle-Phase Turbulence in Gas-Solid Flows, AIChE J. 43 (1997) 853-869.

[17] J. Fessler, J. Eaton, Turbulence modification by particles in a backward-facing step flow, J. Fluid Mech. 394 (1999) 97-117.

[18] D. Koch, Kinetic theory for a monodisperse gas-solid suspension, Physics of Fluids A 2 (1990) 1711-1723.

[19] L. Davidson, An introduction to turbulence models, Tech. Rep. 97/2, Dept. of Thermo and Fluid Dynamics, Chalmers University of Technology, Gothenburg (1997).

[20] Y. Zhang, J. Reese, Gas turbulence modulation in a two-fluid model for gas-solid flows, AIChE J. 49 (2003) 3048-3065.

[21] J. Cao, G. Ahmadi, Gas-particle two-phase turbulent flow in a vertical duct, International Journal of Multiphase Flow 21 (1995) 1203-1228.

[22] M. Sommerfeld, Analysis of collision effects for turbulent gas-particle flow in a horizontal channel: Part I. particle transport, International Journal of Multiphase Flow 29 (2003) 675-699.

[23] P. Johnson, R. Jackson, Frictional-collisional constitutive relations for granular materials, with application to plane shearing, J. Fluid Mech. 176 (1987) 67-93.

[24] H. Versteegh, W. Malalasekera, An Introduction to Computational Fluid Dynamics - The Finite Volume Method, Longman Scientific \& Technical, Harlow, England, 1995. 